\title{
Enhanced Soliton Transport in Quasiperiodic Lattices with Introduced Aperiodicity
}

\author{
Andrey A. Sukhorukov \\ Nonlinear Physics Centre and Centre for Ultra-high bandwidth Devices for Optical Systems (CUDOS), \\ Research School of Physical Sciences and Engineering, Australian National University, Canberra, ACT 0200, Australia
}

(Received 14 July 2005; published 23 March 2006)

\begin{abstract}
We study linear transmission and nonlinear soliton transport through quasiperiodic structures, where the lattice profiles are described by multiple modulation frequencies. We show that resonant scattering at mixed-frequency resonances limits transmission efficiency of localized wave packets, leading to radiation and possible trapping of solitons. We obtain an explicit analytical expression for optimal quasiperiodic lattice profiles, where additional aperiodic modulations suppress mixed-frequency resonances, resulting in dramatic enhancement of soliton mobility. Our results can be applied to the design of photonic waveguide structures, and arrays of magnetic micro-traps for atomic Bose-Einstein condensates.
\end{abstract}

PACS numbers: 42.65.Tg, 03.75.Lm, 42.65.Jx, 42.70.Qs

Periodic structures can provide an efficient control of wave transmission and localization, making it possible to realize new physical regimes that are not allowed in homogeneous systems. Photonic crystals and Bragg gratings are used to tailor dispersion, diffraction, and emission of electromagnetic waves $[1,2]$ and similar concepts have been developed and demonstrated for management of atomic Bose-Einstein condensates in periodic potentials [3,4]. Quasiperiodic photonic structures such as optical superlattices and 2D quasicrystals can offer even more flexibility in designing the properties of optical [5] and atomic matter waves [6,7]. The modulation of quasiperiodic structures is defined by multiple incommensurable spatial frequencies, resulting in the lack of translational symmetry which is a feature of conventional periodic structures. This gives rise to an important effect of stronger field confinement at certain locations due to position-dependent coupling between the lattice sites, which can be utilized for various applications including efficient lasing [8].

Many novel phenomena in periodic structures originate due to the modification of the linear spectrum which is separated into a number of transmission bands and band gaps, where waves experience total reflection [2]. For a one-dimensional lattice with period $d$, the gaps are centered at the frequencies corresponding to Bragg resonance condition for the wave vector component across the lattice, $2 k_{x}=m K$, where $m= \pm 1, \pm 2, \ldots$ is the order of resonance and $K=2 \pi / d$ is the wave number of lattice modulation. In a quasiperiodic structure, primary gaps are defined by several dominant frequencies $K_{j}$; however, there also appear resonances at mixed frequencies $K_{j} \pm$ $K_{l}$, resulting in a complicated spectrum containing multiple mini-bands and mini-gaps [9]. This can have a detrimental effect on the propagation of wave packets through the lattice, which can be inhibited $[6,7]$ or accompanied by strong radiation [10]. It has been predicted that nonlinear wave self-action, which is known to appear due to atomatom scattering in BEC or light-matter interactions in optical crystals, can suppress Anderson localization and allow wave transmission through disordered systems [11]. We find that such nonlinear transmission may be also possible in quasiperiodic lattices in the form of gap solitons (see Refs. [12-17], and references therein). Since the soliton is localized, its spectrum spans over an extended frequency range, and the radiation losses for a moving soliton appear when its spectrum overlaps mixedfrequency resonances. Resolution of these limitations may open new possibilities for realizing efficient transport of optical and matter waves in quasiperiodic structures.

In this Letter, we suggest a novel approach to the design of quasiperiodic structures. We show that mixed-frequency resonances can be suppressed through additional aperiodic lattice modulations which inhibit coherent backscattering at specific frequencies. Aperiodicity is often associated with disorder, leading to the appearance of critically and fully localized states or Anderson localization [7,18]. However, we present an analytical procedure which can be used to define, in a systematic way, quasiperiodic structures with specially introduced aperiodicity possessing regular (absolutely continuous) band-gap spectrum, where all eigenmodes are either extended or exponentially decaying. These "optimal" structures allow for unrestricted motion across the lattice of solitons strongly localized in any of the primary gaps, in full analogy with conventional periodic structures.

We consider below wave transport and soliton motion in effectively discrete systems, such as arrays of optical waveguides or coupled-resonator structures in photonic crystals [19] or arrays of traps for matter waves [20] where the wave transport is defined through tunneling between fundamental modes of the neighboring wells. This process can be described, in the tight-binding approximation, by the discrete nonlinear Schrödinger (or Gross-Pitaevskii) equation for the normalized amplitudes $E_{n}$,

$$
\begin{aligned}
i \frac{\partial E_{n}}{\partial z}+C_{n, n-1} E_{n-1}+ & C_{n, n+1} E_{n+1} \\
& +V_{n} E_{n}+\mathcal{F}\left(n,\left|E_{n}\right|^{2}\right) E_{n}=0 .
\end{aligned}
$$


Here $n$ is the number of waveguide or potential well, $z$ is the propagation distance or time, $C_{n m}$ are coupling coefficients, $V_{n}$ characterizes the detuning between the different sites, and $\mathcal{F}$ defines the nonlinear response $[\mathcal{F}(n, 0) \equiv 0]$. We note that the energy conservation law, $P=\sum_{n}\left|E_{n}\right|^{2}=$ const leads to the requirement that the coupling coefficients are symmetric, $C_{n, n-1}=C_{n-1, n}$. In engineered photonic structures such as waveguide arrays $[15,19]$, there is a flexibility in designing both the on-site potential and also the coupling coefficients. These can be controlled independently by adjusting the waveguide widths and their positions, as schematically illustrated in Figs. 1(a) and 1(b). Recently demonstrated arrays of magnetic micro-traps [21] can be used to precisely design, in a similar way, the trapping potentials for atomic condensates.

The properties of discrete equation (1) with a modulated potential $V_{n}$ but homogeneous coupling $C=$ const were extensively investigated with applications to the problem of electron transport and localization (see Refs. [18,2225], and references therein). It was found that quasiperiodic modulations of $V_{n}$ may lead to Anderson localization [26] and inhibit wave propagation. We show in the following that specially designed modulation of intersite coupling with a constant on-site potential can provide the optimal conditions for linear and nonlinear wave transport in quasiperiodic systems.

We first analyze the characteristic features of wave transport in lattices where the intersite coupling is defined by a superposition of $M$ harmonic modulations, $C_{n, n+1}=$ $\sum_{m=0}^{M} A_{m} \cos \left(K_{m} n+\varphi_{m}\right)$, where $A_{m}, K_{m}$, and $\varphi_{m}$ are free coefficients. For incommensurable modulation frequencies $K_{m}$, the lattice is quasiperiodic, and the Bloch theorem cannot be applied to define the linear spectrum of smallamplitude waves in the form $E_{n ; \beta} \exp (i \beta z)$, where the spectral parameter $\beta$ has the meaning of the propagation
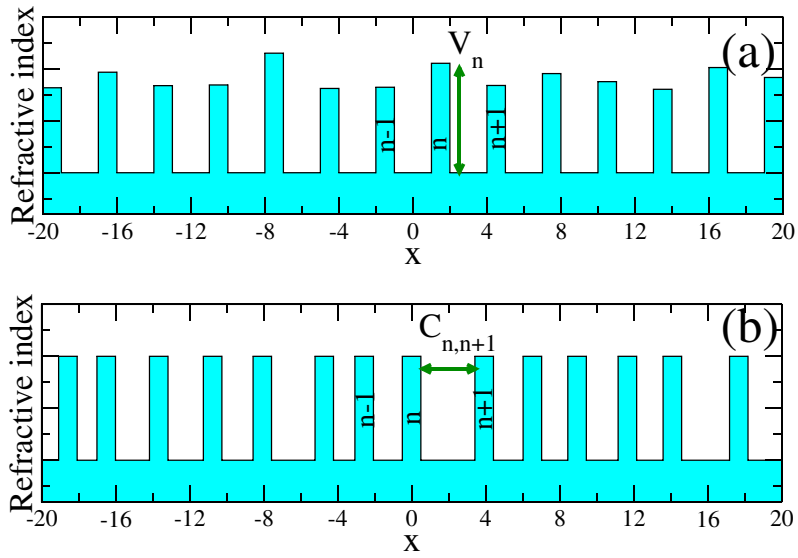

FIG. 1 (color online). Sketch of refractive index (or negative potential) in a lattice with a quasiperiodic modulation of (a) effective on-site potential, proportional to index contrast, and (b) coupling between the lattice sites, inversely proportional to waveguide separation. constant or frequency for optical waves, or negative chemical potential for matter waves. Then, we define the inverse localization length as follows:

$$
\kappa(\beta)=\lim _{n \rightarrow \infty}\left|\frac{2}{n} \log \left[\max _{n / 2<j<n}\left|E_{j ; \beta}\right| / \max _{1<j<n / 2}\left|E_{j ; \beta}\right|\right]\right|,
$$

which we calculate numerically by selecting a sufficiently large computation window to achieve the required accuracy. This dependence defines the linear spectrum: $\kappa \rightarrow 0$ for extended waves inside the transmission bands, and $\kappa>$ 0 correspond to band gaps. The characteristic band-gap spectrum is shown in Fig. 2 (top panel): there is a fundamental gap at large $\beta$, as well as large additional gaps corresponding to Bragg-reflection resonances at the dominant modulation frequencies $K_{m}$. Each of these gaps can support bright solitons in a full analogy with periodic structures [12-17]. We perform numerical simulations and find that such solitons can be excited using the same input conditions as in periodic lattices although some radiation is lost at the input; see Fig. 2 (bottom). Specifically, the input in the form of a tilted Gaussian beam, $E_{n}(z=0)=A_{0} \exp \left(-n^{2} / w^{2}+i k n\right)$, can be used to generate a soliton associated with the fundamental gap, similar to discrete solitons is periodic lattices [14]. The gap solitons are generated using a two-beam $[15,16]$ input, $E_{n}(z=$ $0)=A_{1} \exp \left(-n^{2} / w^{2}+i k_{1} n\right)+A_{2} \exp \left(-n^{2} / w^{2}+i k_{2} n\right)$. Here the beams' inclination angles $\left(k_{j}\right)$ are chosen to match the Bragg resonance at the modulation frequency $K_{m}$,

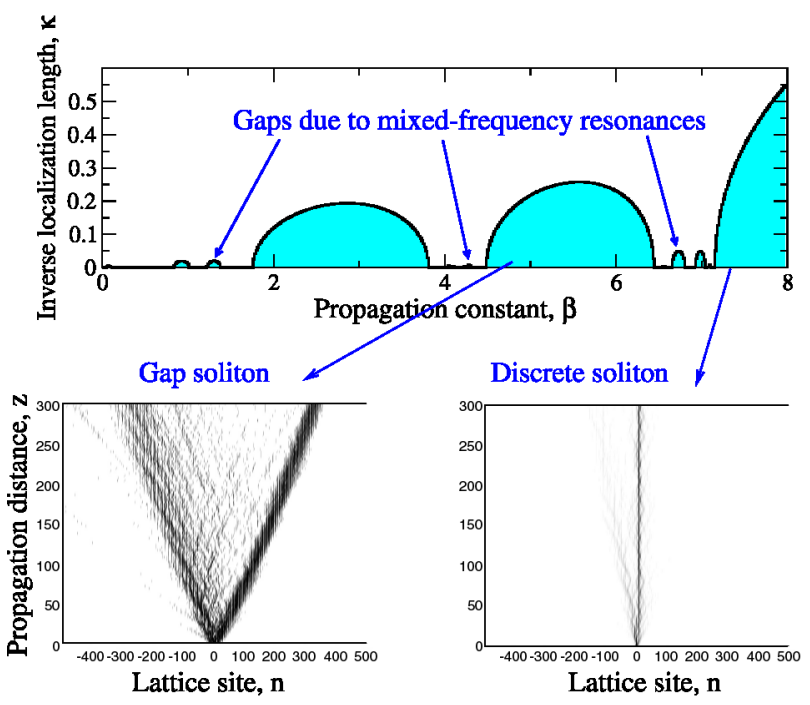

FIG. 2 (color online). Top: Band-gap diagram shown as inverse localization length vs the propagation constant, calculated over 1000 lattice sites. The lattice modulation is given by Eq. (2) with $M=2 ; K_{m} \simeq 0,0.44 \pi, 0.72 \pi ; A_{m} \simeq 3.44,1.02,1.09 ; \varphi_{m} \simeq$ 0, 2.73, 3.42. Bottom: Dynamics of discrete and gap solitons corresponding to the total internal reflection and Braggreflection gaps as indicated by arrows; darker shading corresponds to larger field intensities. Solitons are excited by a single tilted beam with $A_{0} \simeq 0.47, k=0.05 \pi, w=5$ (discrete) or two tilted beams with $A_{1}=A_{2} \simeq 0.27, k_{1}=-0.17 \pi, k_{2}=0.27 \pi$, $w=15$ (gap soliton). 
$\left(k_{2}-k_{1}\right) \simeq K_{m}$, where the inclination of the combined wave front $\left(k_{1}+k_{2}\right)$ can be introduced to induce a soliton motion across the lattice. However, we observe that the presence of mini-gaps strongly affects the wave transport through the lattice. Indeed, the motion of the discrete soliton is arrested as it becomes trapped [Fig. 2 (bottom, right)], similar to effects in disordered systems [27], because the first transmission band (at $6.45<\beta<7.15$ ) is fragmented. The mini-gaps in the second band (at $3.8<$ $\beta<4.45$ ) are weaker, allowing for the motion of the gap soliton [Fig. 2 (bottom, left)]; however, it continuously emits strong radiation during the propagation since its spectrum overlaps the mini-gaps. These simulations are performed for a self-focusing Kerr-type nonlinearity, $\mathcal{F}=$ $\left|E_{n}\right|^{2}$, and similar effects were identified for a defocusing nonlinearity as well.

We now suggest an analytical approach that allows one to calculate the optimal modulation of lattice coupling, where the mixed-frequency resonances are suppressed providing for improved linear and nonlinear wave transport. We note that the model Eq. (1) has an analog in the form of an Ablowitz-Ladik system [28],

$$
i \frac{\partial \psi_{n}}{\partial z}+\left(\psi_{n-1}+\psi_{n+1}\right)\left(C+\left\|\psi_{n}\right\|^{2}\right)=0,
$$

where $\psi_{n}=\left(\psi_{n}^{(1)}, \psi_{n}^{(2)}, \ldots, \psi_{n}^{(M)}\right)$ is a vector function defining the profiles of multiple interacting components, and $\left\|\psi_{n}\right\|^{2}=\sum_{m}\left|\psi_{n}^{(m)}\right|^{2}$. This is considered to be an integrable model [28], where solitons would interact elastically (without radiation losses) with other nonlinear waves, including extended quasiperiodic modes. We find that by identifying the effective modulations of lattice coupling induced by quasiperiodic nonlinear waves in the framework of Eq. (3), we can then define equivalent optimal modulation for the original Eq. (1). Specifically, we consider solutions of Eq. (3) in the form of stationary modulated waves $\psi_{n}^{(m)}(z)=\psi_{n}^{(m)}(0) \exp \left(i \rho_{m} z\right)$, where the profiles $\psi_{n}^{(m)}(0)$ satisfy a set of difference equations,

$$
-\rho_{m} \psi_{n}^{(m)}+\left(\psi_{n-1}^{(m)}+\psi_{n+1}^{(m)}\right)\left(C+\left\|\psi_{n}\right\|^{2}\right)=0 .
$$

The waves with oscillating amplitudes $\psi_{n}$ induce, in the general case, a quasiperiodic lattice with an effective modulation of coupling coefficients, $\tilde{C}_{n, n \pm 1}=C+$ $\left\|\psi_{n}\right\|^{2}$. The lattice modulation can be controlled by choosing the number of modes, the values of $\rho_{m}$, and the initial conditions $\psi_{0}$ and $\psi_{1}$. Dynamics of small-amplitude excitations $\phi_{n} \equiv \psi_{n}^{(M+1)}$ in such lattices is governed by a scalar linear equation with the modulated coupling

$$
i \frac{\partial \phi_{n}}{\partial z}+\left(\phi_{n-1}+\phi_{n+1}\right) \tilde{C}_{n, n \pm 1}=0 .
$$

Most importantly, we observe in numerical simulations that each modulated wave $\psi_{n}^{(m)}$ of Eq. (4) may give rise to a corresponding Bragg-reflection gap for the spectrum of Eq. (5), however there are no gaps at mixed-frequency resonances. We expect that this remarkable property is related to the fact that Eqs. (4) are integrable [29], since the mixed resonances do arise for nonintegrable analogs of Eq. (4). We now introduce a scaling transformation for the mode amplitude, $\phi_{n} \rightarrow \phi_{n} \sqrt{\tilde{C}_{n, n+1}}$, which preserves the structure of the band-gap spectrum and simultaneously transforms Eq. (5) into a linearized Eq. (1) with symmetric coupling found as

$$
\begin{aligned}
C_{n, n+1} & =C_{n+1, n}=\sqrt{\tilde{C}_{n, n+1} \tilde{C}_{n+1, n}} \\
& =\sqrt{\left(C+\left\|\psi_{n}\right\|^{2}\right)\left(C+\left\|\psi_{n+1}\right\|^{2}\right)} .
\end{aligned}
$$

This is the key expression which defines an optimal coupling that can be implemented in real periodic structures.

We show in Fig. 3(a) characteristic quasiperiodic modulation of the intersite coupling coefficient calculated using Eqs. (4) and (6). The Fourier spectrum of the coupling coefficient calculated over 1000 lattice sites contains 5 dominant Fourier components [Fig. 3(b), top], which define the quasiperiodic modulation. However, the bottom plot in Fig. 3(b) demonstrates that there is also a continu-

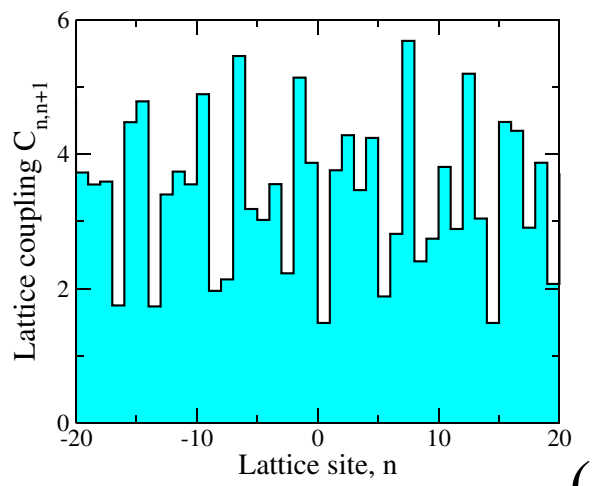

(a)

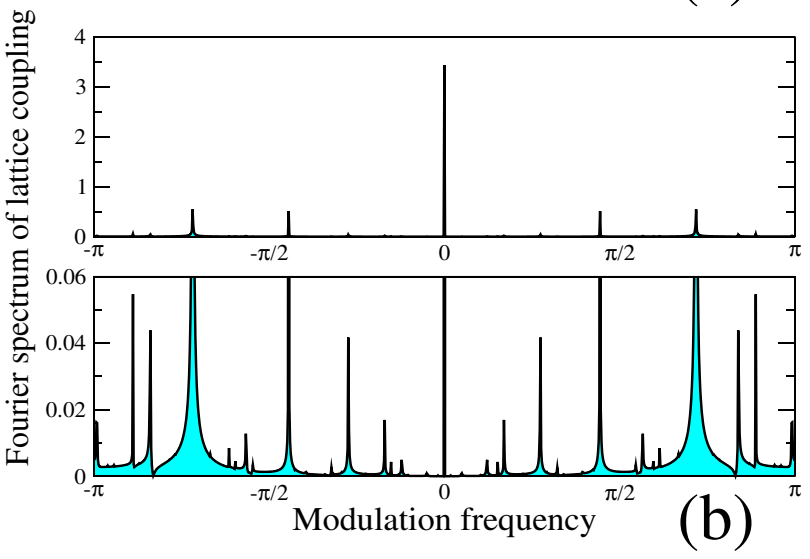

FIG. 3 (color online). Example of a lattice with quasiperiodic coupling and constant on-site potential [as sketched in Fig. 1(b)] calculated with Eqs. (6) and (4): (a) Coupling vs lattice site; (b) Fourier spectrum of the coupling coefficient, shown with different vertical scale in top and bottom plots. Solutions of Eq. (4) are calculated for $\rho_{1}=6.5, \rho_{2}=4, \psi_{0}^{(1)} \simeq 0.51, \psi_{1}^{(1)} \simeq$ $-0.34, \psi_{0}^{(2)} \simeq-0.25, \psi_{1}^{(2)} \simeq 0.75$, and $C=1$. 


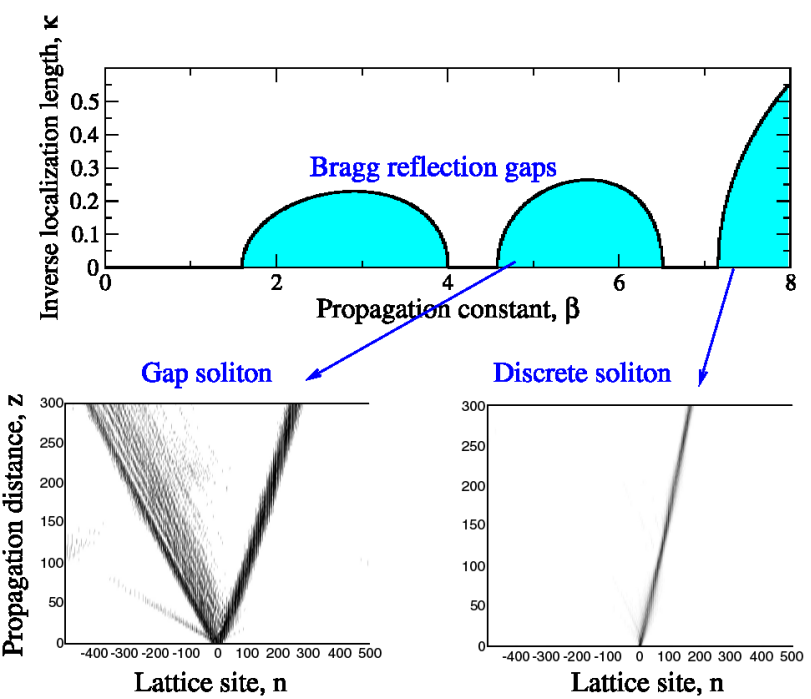

FIG. 4 (color online). Band-gap structure and soliton dynamics in a lattice which modulation corresponds to Fig. 3. Input conditions are the same as in Fig. 2.

ous set of smaller components which describe aperiodic modulations of the lattice. We note that this introduced aperiodicity preserves the long-range order in the lattice. In order to investigate the effect of such modulation, we have chosen the solution parameters for two coupled Eqs. (4) in such a way that dominant peaks in the coupling spectrum [Fig. 3(b), top] exactly correspond to the harmonic modulations used for Fig. 2. Then, we calculate the inverse localization length (Fig. 4, top) and find that the minigaps are absent, in sharp contrast to the band-gap spectrum of lattice with purely harmonic modulations (Fig. 2, top). Moreover, the solitons launched under the same initial condition are now able to propagate through the lattice (Fig. 4,bottom) with a minimal amount of radiation. We note that some radiation is observed at the initial stage due to nonideal input conditions modeling one-beam [14] and two-beam $[15,16]$ experimental arrangements; however, radiation losses of propagating solitons are practically negligible, resembling radiation in periodic lattices [30] that remains exponentially small in a broad parameter region. It is most remarkable that aperiodic modulations defined by Eq. (6) can dramatically improve linear and nonlinear wave propagation and soliton dynamics in quasiperiodic lattices. This is fundamentally different from effects in other types of nonoptimized aperiodic structures such as those defined through modified Thue-Morse sequences, where propagation of fundamental lattice solitons is still accompanied by radiation due to mixed-frequency resonances and gap solitons were not identified [25].

In conclusion, we have demonstrated that linear wave propagation and soliton motion in lattices with exactly quasiperiodic modulation of their parameters can be affected by Bragg resonances at mixed modulation frequencies, leading to soliton trapping or strong radiation losses.
However, these negative effects can be suppressed in lattices with additional aperiodic modulations, and we have presented a systematic analytical method for calculating the parameters of such optimized lattices. These results can be used, for example, to design photonic structures, such as waveguide arrays, and arrays of traps for atomic condensates.

A. S. acknowledges useful discussions with Yuri Kivshar and Costas Soukoulis.

[1] J. D. Joannopoulos et al., Photonic Crystals: Molding the Flow of Light (Princeton University Press, Princeton, 1995).

[2] P. St. J. Russell et al., in Photonic Bloch Waves and Photonic Band Gaps Confined Electrons and Photons, edited by E. Burstein and C. Weisbuch (Plenum, New York, 1995), pp. 585-633.

[3] M. Cristiani et al., Phys. Rev. A 65, 063612 (2002).

[4] T. Anker et al., Phys. Rev. Lett. 94, 020403 (2005).

[5] M. E. Zoorob et al., Nature (London) 404, 740 (2000).

[6] L. Sanchez Palencia and L. Santos, Phys. Rev. A 72, 053607 (2005).

[7] V.W. Scarola and S. Das Sarma, cond-mat/0506415.

[8] M. Notomi et al., Phys. Rev. Lett. 92, 123906 (2004).

[9] J. M. Hollingworth, A. Vourdas, and N. Backhouse, Phys. Rev. E 64, 036611 (2001).

[10] M. Sumetsky et al., Opt. Express 10, 332 (2002).

[11] S. A. Gredeskul and Yu. S. Kivshar, Phys. Rep. 216, 1 (1992).

[12] C. M. de Sterke and J. E. Sipe, in Gap Solitons Progress in Optics Vol. XXXIII, edited by E. Wolf (North-Holland, Amsterdam, 1994), pp. 203-260.

[13] B. J. Eggleton et al., Phys. Rev. Lett. 76, 1627 (1996).

[14] J. W. Fleischer et al., Phys. Rev. Lett. 90, 023902 (2003).

[15] D. Mandelik et al., Phys. Rev. Lett. 92, 093904 (2004).

[16] D. Neshev et al., Phys. Rev. Lett. 93, 083905 (2004).

[17] B. Eiermann et al., Phys. Rev. Lett. 92, 230401 (2004).

[18] C. M. Soukoulis and E. N. Economou, Waves Random Media 9, 255 (1999).

[19] D. N. Christodoulides, F. Lederer, and Y. Silberberg, Nature (London) 424, 817 (2003).

[20] A. Trombettoni and A. Smerzi, Phys. Rev. Lett. 86, 2353 (2001).

[21] A. Guenther et al., Phys. Rev. Lett. 95, 170405 (2005).

[22] S. Aubry and G. Andre, Ann. Isr. Phys. Soc. 3, 133 (1980).

[23] C. M. Soukoulis and E. N. Economou, Phys. Rev. Lett. 48, 1043 (1982).

[24] J. B. Sokoloff, Phys. Rep. 126, 189 (1985).

[25] B. Lindquist, M. Johansson, and R. Riklund, Phys. Rev. B 50, 9860 (1994).

[26] J. Bourgain, Discrete Contin. Dyn. Syst. 10, 75 (2004).

[27] Y. V. Kartashov and V.A. Vysloukh, Phys. Rev. E 72, 026606 (2005).

[28] M. J. Ablowitz et al., Phys. Lett. A 253, 287 (1999).

[29] Yu. B. Suris, Phys. Lett. A 189, 281 (1994).

[30] A. V. Yulin et al., Phys. Rev. Lett. 91, 260402 (2003). 